\title{
Heating the Outer Heliosphere by Pickup Protons
}

\author{
Charles W. Smith*, Philip A. Isenberg*, William H. Matthaeus ${ }^{\dagger}$, John \\ D. Richardson**, Sean Oughton* and Gary P. Zank ${ }^{\S}$ \\ * Space Science Center, University of New Hampshire, Durham, NH 03824 USA \\ ${ }^{\dagger}$ Bartol Research Institute, University of Delaware, Newark, DE 19716 USA \\ ${ }^{* *}$ Massachusetts Institute of Technology, Cambridge, MA 02139 USA \\ ${ }^{\ddagger}$ Department of Mathematics, University of Waikato, Hamilton, New Zealand \\ $\S$ Institute of Geophysics and Planetary Physics, University of California at Riverside, Riverside, \\ CA 92506 USA
}

\begin{abstract}
There is a growing body of literature that demonstrates the ability of a turbulent cascade within the solar wind to heat the thermal protons. Several sources of energy are required to accomplish the observed heating. Wind shear and shocks originating with the multiple source of wind plasma heat the wind inside $\sim 10 \mathrm{AU}$. However, beyond this distance little is left of these sources and all that remains is the energy injected into the plasma by the pickup of newborn protons originating from interstellar neutrals. Recent advances in the theory of wave excitation by the newborn protons allows us to return to the published heating theory and remove a previously unexplained parameterization of the heating due to pickup protons. Furthermore, recent observational evidence suggests that large-scale correlations between the wind speed and the proton temperature exist into the distant outer heliosphere that motivate an attempt to connect the two within the structure of the heating theory.
\end{abstract}

\section{INTRODUCTION}

Measurements by Helios of thermal proton distributions in the inner heliosphere [3,11] reveal ion temperatures hotter than adiabatic expansion would predict. This may not have come as a complete surprise since heating of the ions is responsible for the acceleration of the solar wind [15], and some residual heating might be expected beyond the acceleration point. However, a study of thermal protons measured by the Voyager 2 spacecraft at heliospheric distances from 1 to $42 \mathrm{AU}$ reveals continued heating [17]. By $15 \mathrm{AU}$ the thermal protons are a factor 10 hotter than adiabatic expansion would predict if that prediction is initiated at $1 \mathrm{AU}$ and any heating within that radius is ignored. By $45 \mathrm{AU}$ the discrepancy is nearly a factor of 100 . Beyond $30 \mathrm{AU}$ the temperature of thermal protons is rising with increasing heliocentric distance [20]. Something is heating the solar wind protons in the outer heliosphere.

The expansion of the solar wind is not adiabatic. Two central problems then present themselves: (1) What is the source of the energy? and (2) What is the microphysics that converts the source energy into proton heat? We will set aside question 2 and focus on question 1 exclusively. Question 2 has been addressed by numerous authors. Observational and theoretical insights into the microphysics are consistent with the postulates contained here [4,7-9].

CP719, Physics of the Outer Heliosphere: Third International IGPP Conference, edited by V. Florinski, N. V. Pogorelov, and G. P. Zank

(C) 2004 American Institute of Physics 0-7354-0199-3/04/\$22.00 


\section{THEORY}

The energy source for heating coronal, solar wind, and galactic particles is most often the magnetic field. Dissipation of a static magnetic spectrum of interplanetary waves, where dissipation occurs via cyclotron resonance, provides insufficient energy input to account for the observed heating of thermal protons [19]. Since the observed interplanetary spectrum consistently displays a powerlaw form analogous with the prediction for Navier-Stokes fluid turbulence [6], and since incompressible MHD theory and simulations reproduce this form, we will adopt the view that the interplanetary magnetic field (IMF) fluctuations represent a fundamentally nonlinear evolving turbulent system.

Following observational evidence that the bulk of the energy contained within the inertial range is associated with wave vectors $\mathbf{k}$ perpendicular to the mean field $\mathbf{B}_{\mathbf{0}}$ $[1,2,7,12]$ we construct a theory based on the 2-D turbulent transport of energy by $\mathbf{k} \perp \mathbf{B}_{\mathbf{0}}$ $[13,20,22,23]:$

$$
\begin{aligned}
\frac{d Z^{2}}{d r} & =-\frac{A^{\prime}}{r} Z^{2}-\frac{\alpha}{V_{S W}} \frac{Z^{3}}{\lambda}+\frac{E_{P I}}{V_{S W}} \\
\frac{d \lambda}{d r} & =-\frac{C^{\prime}}{r} \lambda+\frac{\beta}{V_{S W}} Z-\frac{\beta}{V_{S W}} \frac{\lambda}{Z^{2}} E_{P I} \\
\frac{d T}{d r} & =-\frac{4}{3} \frac{T}{r}+\frac{1}{3} \frac{m_{p}}{k_{B}} \frac{\alpha}{V_{S W}} \frac{Z^{3}}{\lambda}
\end{aligned}
$$

where $Z^{2}$ is the fluctuation energy in Elsasser variables, $\lambda$ is the similarity length scale, $T$ is the proton temperature, $r$ is the heliocentric distance, $V_{S W}$ is the solar wind speed, $m_{p}$ is the proton mass, and $k_{B}$ is Boltzmann's constant. $A^{\prime}=-1.1, C^{\prime}=1.8$ are constrained by solar wind conditions and $\alpha=1=\beta$ are constrained by Taylor-Karman local phenomenology. $E_{P I}$ is the driving term due to waves originating from newborn interstellar pickup protons.

The above formalism assumes that the energy available for heating the plasma is whatever amount of energy is transported through the inertial range by the turbulent cascade from the large-scales to the small-scales. In this way, it is the transport rate that governs the dissipation rate and not the detailed dissipation processes. Three energy sources are contained within the above equations: turbulence driven by wind shear, shock heating, and waves due to pickup protons. Wind shear and shock heating possess similar scalings and are absorbed into the terms containing $A^{\prime}$ and $C^{\prime}$. Energy production by pickup protons is contained within $E_{P I}$.

Our original form for $E_{P I}$ is [21]:

$$
E_{P I}=\frac{d n_{P I}}{d t} \frac{V_{A} V_{S W}}{n_{S W}}
$$

where $N_{P I}$ is the density of pickup protons, $t$ is time, and $V_{A}$ is the local Alfvén speed. This is the form that assumes particle scattering onto bispheres separated by twice the local Alfvén speed. The production rate for newborn pickup protons is given by:

$$
\frac{d n_{P I}}{d t}=\frac{n_{S W}^{0}}{\tau_{i o n}^{0}}\left(\frac{r_{E}}{r}\right)^{2} e^{-\lambda_{P I} \theta / r \sin (\theta)}
$$


where $n_{S W}^{0}$ is the density of solar wind thermal protons at $1 \mathrm{AU}$ which is typically $5 \mathrm{~cm}^{-3}, \tau_{i o n}^{0}$ is the neutral ionization time at $1 \mathrm{AU}$ which we take to be $10^{6} \mathrm{~s}, \lambda_{P I}$ is the ionization cavity length scale which we take to be $8 \mathrm{AU}$ (interstellar neutral $\mathrm{H}$ does not penetrate inside $8 \mathrm{AU}$ prior to ionization by either photoionization or collision with a solar wind ion), $r / r_{E}$ is heliocentric distance in units of $\mathrm{AU}$, and $\theta$ is the spacecraft heliographic latitude (interstellar neutrals are assumed to enter at $0^{\circ}$ ). Some authors prefer a smaller $\lambda_{P I} \sim 5 \mathrm{AU}[5,18]$. The resulting expression for energy injection by newborn pickup protons is:

$$
E_{P I}=\frac{V_{S W}}{n_{S W}^{0}} \frac{V_{A} n_{H}^{\mathrm{inf}}}{\tau_{i o n}^{0}} e^{-\lambda_{P I} \theta / r \sin (\theta)}
$$

where $n_{H}^{\text {inf }}$ is the density of $\mathrm{H}$ outside the heliosphere. This term greatly exceeds the observed heating rate, requiring introduction of a parameter $[20,22]$

$$
E_{P I} \rightarrow f_{D} E_{P I}
$$

where the value $f_{D}=0.08$ yields good agreement with the observations. The physical explanation for $f_{D}$ was not clear at the time. However, it was readily apparent that $f_{D}=1$, whereby the full amount of energy made available by scattering onto the bisphere was converted into heat, produced a solar wind that was an order of magnitude hotter than observed.

The theory of pickup proton scattering and wave generation is recast [5] by including three advances in the theory and making one assumption: (1) Wave dispersion in the ion/wave resonance is included, (2) Scattering by both Alfvén and fast mode waves is included, (3) Broadening of the newborn distribution due to IMF fluctuations is included, and (4) Maintenance of a powerlaw spectrum of magnetic fluctuations is assumed. The first three conditions are natural advances in kinetic theory. The last is motivated by the observed reproducibility of the powerlaw spectrum and assumes implicitly that whatever wave energy is generated by newborn protons is subsequently transported through the spectrum by incorporation into the spectral cascade and does not accumulate. This is substantially different from previous descriptions [10], but consistent with the observed waves due to pickup protons [14]. The revised formalism [5] modifies the contour on which newborn pickup protons scatter so that it is more nearly spherical than bispherical. This modification results in less energy production in the form of waves and validates the earlier approximate solution [20] using $f_{D}=0.08$, although such an approximation misses some variability.

\section{APPLICATION OF THEORY TO OBSERVATIONS}

Our earlier effort to apply the theory to observations [20] renormalized the observations in the outer heliosphere using $1 \mathrm{AU}$ measurements recorded on the Omnitape data set in an effort to remove variability of the solar source. The measured variation of $Z^{2}$ and $T$ at $1 \mathrm{AU}$ were used to rescale the observations by Voyager 2 and Pioneer 11 . However, the theory also depends sensitively on $V_{S W}$ and $V_{A}$. We did not attempt to relate variations in 
the observed $T$ to variations in the other bulk parameters that drive the theory. Instead, the theory used a single set of parameters to put smooth curves through the observations. The agreement was good, subject to variation of $f_{D}$, but there remained the question of whether the variation in the observations resulted from statistical fluctuations or could be reproduced in detail by the theory using the observed variation of the bulk parameters. However, the temperature variations are closely linked to variations in the wind speed [16]. This means that a more detailed application of the theory should be able to reproduce the observed variation of the proton temperature and link that to the observed variation in the wind and Alfvén speeds.

To do this, we start with the observations at 1 AU. We discard the process of normalizing the measurements of the outer heliosphere using the $1 \mathrm{AU}$ results and input the 1 AU measurements directly into the theory. The 1 AU measurements are averaged over an integer number of solar rotations so that some statistical weight is obtained and the issue of longitudinal separation of Earth and the spacecraft of the outer heliosphere can be ignored. The theory is then evolved outward to the location of the spacecraft and the resulting prediction for the magnetic fluctuation energy and proton temperature is compared directly with the observed values. The theory used is the same as earlier [20] with the parameter $f_{D}=0.08$ justified separately [5].

Figure 1 shows the results of this analysis. While the theory explains the general trend in magnetic fluctuation energy (top panel), it is unable to account for observed variability. Using higher time resolution data would probably raise the observational values to be more in keeping with the theory. It is a feature of this theory that elevated levels in magnetic energy are subject to increased spectral transport and enhanced energy dissipation. The temperature prediction (2nd panel) follows the observed trends very closely. It catches the rapid rise at $25 \mathrm{AU}$, but fails to reproduce the observations from 45 to $58 \mathrm{AU}$. The reason for that discrepancy is readily seen. The spacecraft latitude (3rd panel) is rapidly descending into the southern latitudes. This occurs at solar minimum when the solar wind conditions below $30^{\circ}$ south latitude are distinctly different from the input values recorded by Omnitape. Simply put, Omnitape is an inappropriate input for comparison with Voyager at this time. This interpretation is confirmed by the ratio of the Voyager and Omnitape mean wind speeds (bottom panel). Voyager sees significantly higher wind speeds at this same time, in keeping with the assertion that the observed discrepancy in temperature is a latitudinal effect created by solar minimum conditions. As the wind speed observed at Voyager returns to agreement with the observations of Omnitape, the disagreement in proton temperature decreases and the theory converges with the observations.

\section{SUMMARY}

We have demonstrated that the turbulent transport theory described here can predict the observed temperature of solar wind protons using bulk parameters when suitably averaged over the time scale of a few months. Interstellar newborn pickup protons act as the primary energy source for heating the thermal wind in the outer heliosphere. Statistical fluctuations of the observed quantities that result from inadequate sampling 


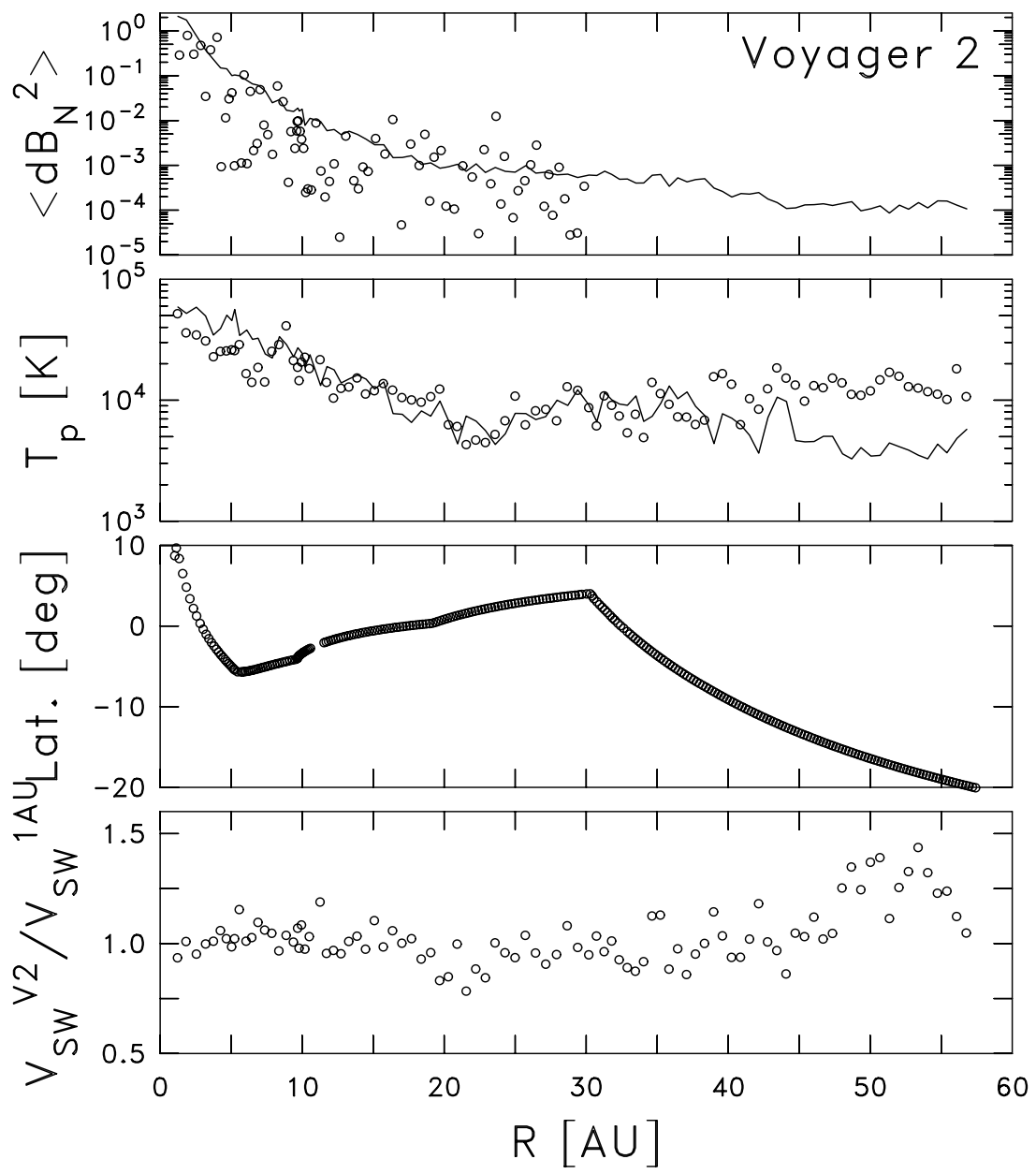

FIGURE 1. Comparison of Voyager 2 observations with prediction of theory using Omnitape as input. (top panel) Measured magnetic fluctuation level plotted as circles (N-component only) and prediction. Prediction is at high range of observations, perhaps due to use of 1-hr data. (panel 2) Observed proton temperatures compared with theoretical prediction. Note discrepancy from 45 to $58 \mathrm{AU}$. This corresponds to solar minimum. (panel 3) Heliocentric latitude of Voyager. Note far southern excursion from $45 \mathrm{AU}$ onward. (bottom panel) Comparison of average wind speed at Voyager with $1 \mathrm{AU}$ average. Note increased wind speed in outer heliosphere from 45 to $58 \mathrm{AU}$ corresponding to solar minimum conditions.

appear to be comparable to the difference between the observed and predicted values of the thermal proton temperature and is smaller than the systematic variations of the energy sources to the degree they are accurately resolved. In other words, this theory can describe the heating of the solar wind down to the scale of monthly variations so long as the bulk parameters of the wind are known and adequately represented by single 
spacecraft measurements.

\section{ACKNOWLEDGMENTS}

Support at the University of New Hampshire is derived from NASA GI grant NNG04GA24G and the NASA Sun-Earth Connection Theory Program under grant NAG5-11797. Support at the University of Delaware is derived from ATM-0105254. Support at the Massachusetts Institute of Technology is derived from JPL subcontract 959203. Support at the University of Waikato is derived from the NZ Marsden fund (02-UOW-050 MIS). Support for the University of California at Riverside is derived from NASA grant NAG5-12903.

\section{REFERENCES}

1. Bieber, J. W., W. H. Matthaeus, C. W. Smith, W. Wanner, M.-B. Kallenrode, and G. Wibberenz, Astrophys. J., 420, 294-306, 1994.

2. Bieber, J. W., W. Wanner, and W. H. Matthaeus, J. Geophys. Res., 101, 2511-2522, 1996.

3. Freeman, J. W., Geophys. Res. Lett., 15, 88-91, 1988.

4. Gary, S. P., and J. Borovsky, J. Geophys. Res., in press, 2004.

5. Isenberg, P. A., C. W. Smith, and W. H. Matthaeus, Astrophys. J., 592, 564-573, 2003.

6. Kolmogoroff, A. N., Dokl. Akad. Nauk SSR, 30, 301-305, 1941.

7. Leamon, R. J., C. W. Smith, N. F. Ness, W. H. Matthaeus, and H. K. Wong, J. Geophys. Res., 103, 4775-4787, 1998a.

8. Leamon, R. J., W. H. Matthaeus, C. W. Smith, and H. K. Wong, Astrophys. J., 507, L181-L184, 1998 b.

9. Leamon, R. J., C. W. Smith, N. F. Ness, and H. K. Wong, J. Geophys. Res., 104, 22,331-22,344, 1999.

10. Lee, M. A., and W.-H. Ip, J. Geophys. Res., 92, 11,041-11,052, 1987.

11. Marsch, E., in Physics of the Inner Heliosphere, Vol. 2, Particles, Waves and Turbulence, edited by R. Schwenn and E. Marsch, pp. 45-133, Springer-Verlag, New York, 1991.

12. Matthaeus, W. H., M. L. Goldstein, and D. A. Roberts, J. Geophys. Res., 95, 20,673-20,683, 1990.

13. Matthaeus, W. H., G. P. Zank, C. W. Smith, and S. Oughton, Phys. Rev. Lett., 82(17), 3444-3447, 1999.

14. Murphy, N., E. J. Smith, B. T. Tsurutani, A. Balogh, and D. J. Southwood, Space Sci. Rev., 72 (1-2), 447-453, 1995.

15. Parker, E. N., Interplanetary Dynamical Processes, Wiley-Interscience, New York, 1963.

16. Richardson, J. D., and C. W. Smith, Geophys. Res. Lett., 30(5), 1206, doi:10.1029/2002GL016551, 2003.

17. Richardson, J. D., K. I. Paularena, A. J. Lazarus, and J. W. Belcher, Geophys. Res. Lett., 22, 325-328, 1995.

18. Rucinski, D., A. C. Cummings, G. Gloeckler, A. J. Lazarus, E. Möbius, and M. Witte, Space Sci. Rev., 78, 73, 1996.

19. Schwartz, S. J., W. C. Feldman, and S. P. Gary, J. Geophys. Res., 86, 541-546, 1981.

20. Smith, C. W., W. H. Matthaeus, G. P. Zank, N. F. Ness, S. Oughton, and J. D. Richardson, J. Geophys. Res., 106, 8253-8272, 2001.

21. Williams, L. L., G. P. Zank, and W. H. Matthaeus, J. Geophys. Res., 100, 17 0599-17067, 1995.

22. Zank, G. P., W. H. Matthaeus, and C. W. Smith, J. Geophys. Res., 101, 17,093-17,107, 1996.

23. Zhou, Y., and W. H. Matthaeus, J. Geophys. Res., 95, 10,291-10,311, 1990. 